\title{
Factor and Cluster Analysis for TCM Syndromes of Real-World Metabolic Syndrome at Different Age Stage
}

\author{
Shujie Xia (D), ${ }^{1,2}$ Jing Cai, ${ }^{3}$ Jianxin Chen ${ }^{D},{ }^{4}$ Xuejuan Lin, ${ }^{1,2}$ Shujiao Chen, ${ }^{1,5}$ \\ Bizhen Gao $\mathbb{D}^{1},{ }^{1,3}$ and Candong $\operatorname{Li} \mathbb{i}^{1,2}$ \\ ${ }^{1}$ Syndrome Research Base of Traditional Chinese Medicine, Fujian University of Traditional Chinese Medicine, \\ Fuzhou 350122, China \\ ${ }^{2}$ Fujian Key Laboratory of TCM Health State, Fuzhou 350122, China \\ ${ }^{3}$ College of Integrative Chinese and Western Medicine, Fujian University of Traditional Chinese Medicine, Fuzhou 350122, China \\ ${ }^{4}$ College of Traditional Chinese Medicine, Beijing University of Traditional Chinese Medicine, Beijing 100105, China \\ ${ }^{5}$ The Third People's Hospital of Fujian Province, Fuzhou 350108, China
}

Correspondence should be addressed to Bizhen Gao; gbz688@163.com and Candong Li; warm_wind888@163.com

Received 19 February 2020; Accepted 2 June 2020; Published 8 July 2020

Academic Editor: George B. Lenon

Copyright (c) 2020 Shujie Xia et al. This is an open access article distributed under the Creative Commons Attribution License, which permits unrestricted use, distribution, and reproduction in any medium, provided the original work is properly cited.

Background. Traditional Chinese medicine (TCM) has the characteristics of multitarget and overall regulation, which has certain advantages in preventing and treating of metabolic syndrome (MS). The aim of the present study was to evaluate the similarities and differences of TCM syndrome distribution in different age groups to promote the optimization of treatment strategies. Methods. This study was based on a real-world survey conducted in 3 hospitals in China. There are 1262 collected cases of MS meeting the inclusion criteria, which were divided into the young group, middle-aged group, and elderly group. Factor analysis (FA) was conducted for syndrome element extraction, and $K$-means cluster analysis was processed for syndrome type classification. Frequency analysis and Chi-square test were used to compare the syndrome characteristics of each group. Results. Common factors extracted were assigned to 18 syndrome elements including 11 nature syndrome elements and 7 location syndrome elements. Phlegm and dampness are the most frequent syndrome elements in general. Compared with the middle-aged group and elderly group, the young group has more obvious nature syndrome elements in heat and Qi deficiency, and location syndrome elements in the stomach. As for the middle-aged group, the frequency of location syndrome in kidney syndrome was higher than that in other groups. When it comes to the elderly group, it is shown that the symptoms of Yin deficiency and blood deficiency increase with age and the old patients may have more location syndrome elements in the lung and gallbladder. Conclusion. The TCM syndrome of MS is complex in that there may be the characteristics of deficiency and excess syndrome simultaneously. The main pathological factors of MS were phlegm and dampness. Besides, MS patients at different age periods may have their own syndrome distribution features. So, it is reasonable to adhere to the method of resolving phlegm and removing dampness and, at the same time, adopt the ways of clearing heat, promoting Qi, nourishing Yin, supplementing blood as well as regulating the liver, promoting the lung, transporting the spleen, nourishing the heart, and nourishing the kidney based on the syndrome feature of MS in different age stages.

\section{Introduction}

Metabolic syndrome (MS) is a metabolic disorder syndrome characterized by obesity, hyperglycemia, hypertension, dyslipidemia, and hyperuricemia, which are the pathological basis of cardiovascular and cerebrovascular diseases and diabetes [1]. Studies have shown that the incidence of MS increases with age, and the earlier the age of onset, the greater the risk of disease [2-4]. At present, the incidence of MS is $58.1 \%$ in the population over 60 years old in China, which seriously endangers people's health [5]. Traditional Chinese medicine(TCM) has the characteristics of multitarget, small side effects and overall regulation. There are many studies showing that the therapeutic effects of MS in 
TCM are fairly satisfying [6-8]. In TCM clinical practice, syndrome differentiation serves as the core of treatment for MS [9]. When differentiating a patient's syndrome pattern, the practitioner systematically collects comprehensive information about the presenting signs and symptoms by the four diagnostic methods of inspection, auscultation/smelling, interrogation, and palpating. Then, the collocated information is evaluated according to the TCM theory and clinical experience to identify the pathological characteristics of the current stage and differentiate the syndrome pattern. After that, TCM treatment will be applied in accordance with the conclusions drawn from the syndrome differentiation process.

Related studies focused on syndrome classification based on data mining methods. Various classification techniques, e.g., artificial neural network, naïve bayes, $k$-nearest neighbor, and multilabel learning were applied in quantitative syndrome analysis [10-13]. Better results were obtained for several important diseases, e.g., coronary heart disease, diabetes, and viral hepatitis. By introducing these data mining models, researchers can mine the objective syndrome feature distribution for diseases from some effective cases. Such feature distribution can be used to characterize the underlying relationship between the current disease status and its specific symptoms. There are some studies explore the overall syndrome distribution characteristics of MS or related diseases [14, 15]. However, few studies explored the underlying syndrome features in MS at different age stages. Even though it is the same disease, the treatment methods are different according to the syndrome characteristics of different age stages. So, it is of great significance to determine the syndrome features of MS at different age stages from effective clinical cases.

In this study, we use the multivariate statistical method to explore the distribution feature of TCM syndrome in MS patients at different age stages who really need a physical examination or TCM treatment. First, factor analysis (FA) was applied to reduce the dimensionality of a large number of TCM symptoms and signs, by which these TCM variables can be remodeled as linear combinations of a smaller number of underlying factors. Second, according to the underlying factors, i.e., common factors, the TCM syndrome elements were further extracted in combination with professional knowledge. Third, the distribution of TCM syndrome elements in different age groups was compared and analyzed based on factor scores. Then, the common factor scores of each patient were included as variables in the cluster analysis, which could find the potential combination rule of each factor and then classify each sample. Lastly, we summarized the main TCM syndromes types contained in each category and analyzed the distribution feature of syndrome types in different age groups. In this way, better TCM syndrome features can provide objective diagnostic evidence for clinical doctors to improve the accuracy of syndrome diagnosis and efficacy of clinical treatment for MS.

This paper consists of the following sections. Materials and Methods introduce case selection and analysis methods. Results show the syndrome distribution outcomes of MS in different age groups.
Discussion and conclusion analyze the reasons for the difference of syndrome distribution in different groups, and the limitations of this research and future works are also discussed.

\section{Materials and Methods}

2.1. Study Design and Participants. The inpatients and outpatients with MS were selected in the Second People's Hospital Affiliated to the Fujian University of TCM, the Third People's Hospital Affiliated to the Fujian University of TCM, the Fuzhou Second Hospital, and the Jinjiang Hospital of TCM from November 2013 to October 2019. The participants were divided into three groups according to the following criteria: young group (YG, 18-39 years old), middle-aged group (MG, 40-59 years old), and elderly group (EG, 60 years old and above). All participants signed consent forms. Ethics approval for the present study was given by the Medical Ethics Committee of the Fujian University of Traditional Chinese Medicine.

2.2. Sample Size Estimation. According to the number of four diagnostic information items that may appear in MS patients of all age groups and the principle of "the number of samples must be $5-10$ times the number of variables," the sample size was calculated considering the $10 \%$ missing rate. In this study, there were 45 symptom variables with a frequency of more than $10 \%$, to ensure the accuracy of the study to the greatest extent, and the total sample size of the study was 1262 .

2.3. Diagnostic Criteria. Diagnostic criteria of Western medicine were as follows: according to the MS diagnostic criteria issued by the IDF, AHA, and Diabetes Society of the Chinese Medical Association, the diagnosis can be made if the following 3 items or more are met: (1) abdominal obesity (waist circumference: male $\geq 90 \mathrm{~cm}$ and female $\geq 85 \mathrm{~cm}$ ); (2) hyperglycemia: $\mathrm{FPG} \geq 6.1 \mathrm{mmol} / \mathrm{L}$ or $2 \mathrm{hPG} \geq 7.8 \mathrm{mmol} / \mathrm{L}$ and/or diagnosed as diabetes; (3) hypertension: $\mathrm{BP} \geq 130$ / $85 \mathrm{mmHg}$ and/or diagnosed as hypertension; (4) $\mathrm{TG} \geq 1.70 \mathrm{mmol} / \mathrm{L}$; and (5) HDL-C $<1.04 \mathrm{mmol} / \mathrm{L}$. TCM syndrome element and type diagnosis standard was as follows: according to the Syndrome Part of TCM Clinical Diagnosis and Treatment Terminology (the National Standard of the People's Republic of China) [16], TCM Diagnostics [17], Syndrome Elements Differentiation [18] and combined with the expert consultation, the classification specification of syndrome patterns was formulated.

2.4. Inclusion and Exclusion Criteria. Inclusion criteria of the patients are (1) patients who meet the diagnostic criteria of MS; (2) patients who are given the informed consent; and (3) patients' age range from 18 to 75 .

Exclusion criteria are (1) patients with mental diseases or other severe diseases; (2) patients who could not express their feelings clearly; and (3) patients who refuse to participate in our study or without informed consent. 
2.5. Analysis Method. According to the "syndrome differentiation significance of 600 common symptoms" and the MS common symptom standard in the Guideline of Clinical Research of TCM New Drugs, the four diagnosis information collection scale is established. The symptoms and signs were classified as none, mild, moderate, and severe with $0,1,2$, and 3 points, respectively. For symptoms and signs that are difficult to be graded, they were classified into nonoccurrence and occurrence with 0 and 1 points, respectively.

SPSS 24.0 software was used for statistical processing and analysis. Factor analysis was processed by the KMO test, Bartlett spherical test, and principal component analysis, and then the distribution of nature and location syndrome elements in different age groups was obtained. On the basis of factor analysis, the K-means clustering method was used to analyze the distribution of syndrome types in different age groups (Figure 1). The Chi-square test was used for the comparison of counting data, and the Bonferroni method was used for the comparison between two groups to correct the inspection level. The corrected inspection level $\alpha=(0.05 / 3)$ $=0.0167$. All data were entered, counted, and processed by professionals.

2.6. Quality Control. The study was carried out with strict quality control. All investigators were specialized in TCM or integrated Chinese and Western medicine and trained in the standard operating procedures for the study. Each study participant was examined and followed up by at least two resident physicians or graduate students who filled in the CRFs. At least two senior staff physicians supervised the interview sessions to ensure consistency and authenticity of data collection to reduce measurement bias.

\section{Result}

3.1. General Result. In this study, 1262 cases of MS were collected (Table 1), including 554 males (43.90\%) and 708 females $(56.10 \%)$, with an average age of 51 years. There were 209 cases (16.56\%) in the young group, 131 males and 78 females, with an average age of 33 years. There were 740 cases (58.64\%) in the middle-aged group, 299 males and 441 females, with an average age of 50 years. There were 313 patients $(24.80 \%)$ in the elderly group, 124 males and 189 females, with an average age of 65 years.

\subsection{Frequency Analysis of Four Diagnostic Information.} The four diagnosis information of MS patients in different age groups was tabulated based on the distribution frequency. Each group screened the first 20 symptoms and signs (Table 2).

\subsection{Factor Analysis of Syndrome Elements}

3.3.1. Suitability Test. The KMO test and Bartlett's test of sphericity were used to evaluate the suitability of collocated diagnostic variables for factor analysis. The Kaiser-Meyer-Olkin (KMO) test assesses the partial correlation between variables, and if the $\mathrm{KMO}$ value is $>0.5$, the variable will be more suitable for factor analysis. In addition, the closer the KMO value is to 1 , the stronger the correlation is between variables. Bartlett's test of sphericity assesses the null hypothesis and whether the correlation matrix is rejected as a unit matrix. Only when the variables are relatively nonindependent $(P<0.05)$, they can be used for factor analysis.

In this study, the KMO value of the partial correlation of variables was $0.627>0.5$, indicating a certain degree of partial correlation between variables and factor analysis could be carried out. The approximate Chi-square value of Bartlett's test of sphericity was 8403.438 , and $P<0.001$, indicating a strong correlation between variables and rejection of the hypothesis of independence of variables. So, the variables could be applied to factor analysis.

3.4. Extraction Factors. Characteristic root value is an index to evaluate the influence of the extracted common factors, which indicates how much the information of the original variables a common factor can explain. The cumulative variance contribution rate (CVCR) is the accumulation of variance contribution rate of the first $N$ common factors, which shows the proportion of information of the first $N$ common factors is covered. A total of 45 variables of symptoms and signs with a frequency of more than $10 \%$ are included in the factor analysis. The principal component analysis was applied to extract common factors. The characteristic root values of the first 19 common factors were no less than 1 , and their CVCR reached $62.06 \%$. From the scree plot (Figure 2), we can see the relevance of common factors and characteristic root values. The scatter locations of the first 19 common factors were steep, which means the first 19 common factors bear the most information in this research.

3.5. Factor Rotation and Transformation. The purpose of factor rotation is to allow the factor load the absolute value of the new common factor for each of the variables to polarize to 0 or 1 . It can facilitate the naming and interpretation of the main factors in this study. After the maximum rotation of variance and setting 0.3 as the threshold value of the load coefficient, each common factor was allocated to the most relevant index. Based on the results of factor rotation and combined with professional knowledge, we summarized 18 syndrome elements (Table 3), including 11 nature syndrome elements and 7 location syndrome elements. The nature syndrome elements include phlegm, dampness, heat, cold, Qi stagnation, blood stasis, Yang hyperactivity, Yin deficiency, Yang deficiency, blood deficiency, and Qi deficiency, respectively. The location syndrome elements include the heart, lung, liver, spleen, kidney, stomach, and gallbladder.

3.6. Common Factor Equation for Syndrome Elements. Through the common factor equation, the scores of each patient on each of the 19 common factors were obtained. Then, the distribution of the syndrome elements in each group was analyzed according to the highest score. There were obvious differences in the overall distribution of nature 


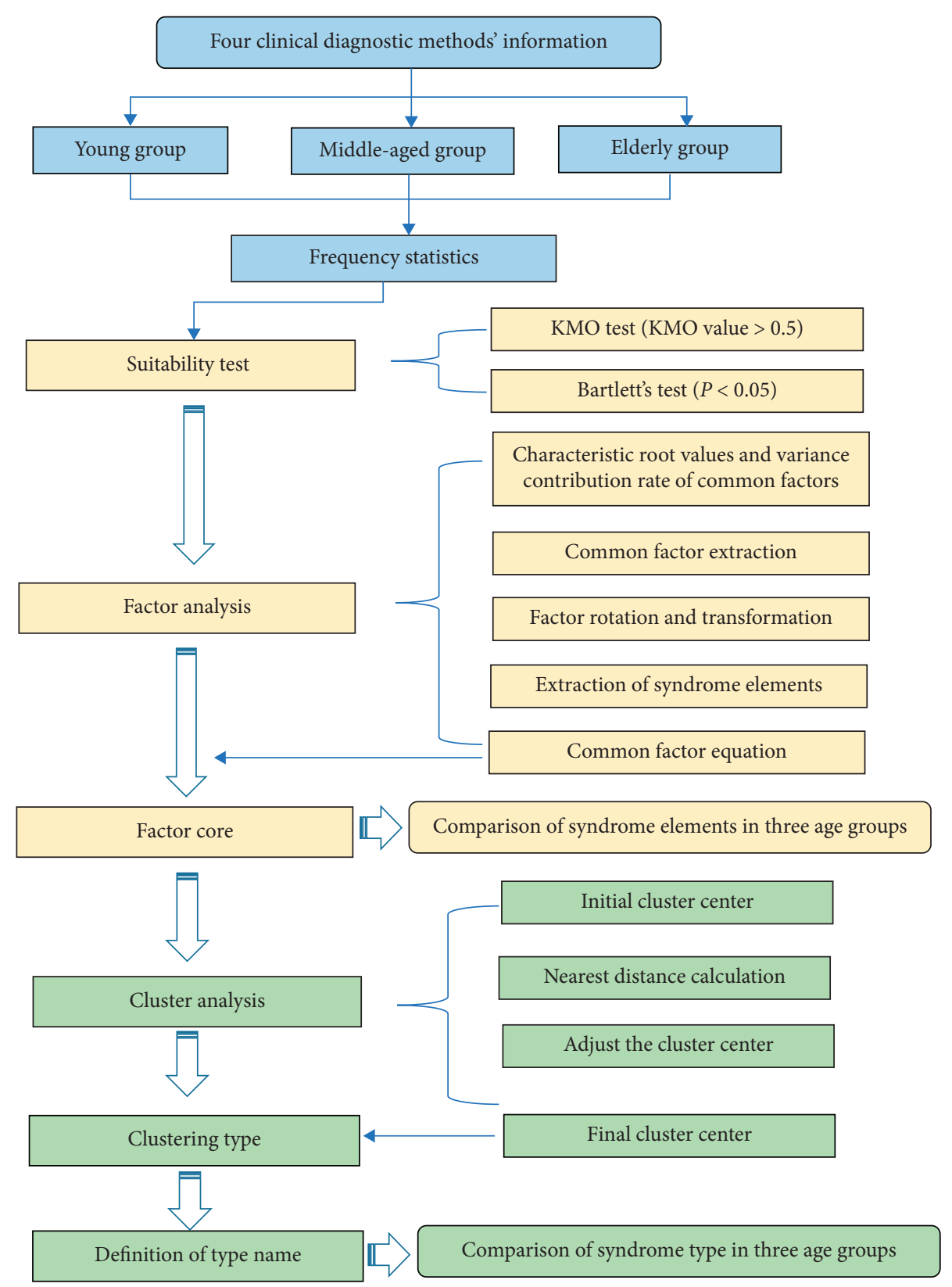

FIGURE 1: Flowchart of factor and cluster analysis-based MS syndrome pattern extraction. First, TCM clinical information of MS patients in different age groups was collected. Then, the collected variables were dimensionally reduced by factor analysis, and the main syndrome elements of each group were extracted. Finally, cluster analysis was used to classify the syndrome elements, so as to further summarize the distribution characteristics of MS syndrome types.

TABle 1: Age and sex distribution of MS patients in three groups.

\begin{tabular}{|c|c|c|c|c|c|c|c|}
\hline \multirow{2}{*}{ Group } & \multirow{2}{*}{ Average age } & \multicolumn{2}{|c|}{ Male } & \multicolumn{2}{|c|}{ Female } & \multicolumn{2}{|c|}{ Total } \\
\hline & & $N$ & $\%$ & $N$ & $\%$ & $N$ & $\%$ \\
\hline Young group & 33 & 131 & 62.68 & 78 & 37.32 & 209 & 16.56 \\
\hline Middle-aged group & 50 & 299 & 40.41 & 441 & 59.59 & 740 & 58.64 \\
\hline Elderly group & 65 & 124 & 39.62 & 189 & 60.38 & 313 & 24.80 \\
\hline Total & 51 & 554 & 43.90 & 708 & 56.10 & & \\
\hline
\end{tabular}

and location syndrome elements in the three different age groups $(P<0.001)$ by the $X^{2}$ test.

In the aspect of nature syndrome elements (Table 4, Figure 3 ), the top three frequencies in the young group were phlegm, heat, and dampness syndrome; in the middle-aged group, the top three frequencies were phlegm, dampness, and heat syndrome; and in the elderly group, the top three frequencies were dampness, phlegm, and Yin deficiency 
TABLE 2: Frequency statistics of four diagnostic information.

\begin{tabular}{|c|c|c|c|c|c|c|c|c|c|}
\hline \multirow{2}{*}{ Ranking } & \multicolumn{3}{|l|}{ Young group } & \multicolumn{3}{|c|}{ Middle-aged group } & \multicolumn{3}{|c|}{ Elderly group } \\
\hline & Variables & $N$ & $\%$ & Variables & $N$ & $\%$ & Variables & $N$ & $\%$ \\
\hline 1 & Wiry pulse & 89 & 42.58 & Wiry pulse & 378 & 51.08 & Wiry pulse & 197 & 62.94 \\
\hline 2 & Slippery pulse & 87 & 41.63 & Thirsty & 339 & 45.81 & Thirsty & 151 & 48.24 \\
\hline 3 & Thirsty & 85 & 40.67 & Greasy tongue coating & 287 & 38.78 & Greasy tongue coating & 131 & 41.85 \\
\hline 4 & Greasy tongue coating & 83 & 39.71 & Slippery pulse & 281 & 37.97 & Slippery pulse & 120 & 38.34 \\
\hline 5 & Pulse sinking & 70 & 33.49 & Bitter taste & 225 & 30.41 & Dizzy & 119 & 38.02 \\
\hline 6 & Tooth-marked tongue & 59 & 28.23 & Dizzy & 217 & 29.32 & Bitter taste & 118 & 37.70 \\
\hline 7 & Thready pulse & 57 & 27.27 & Acid heavy pain & 211 & 28.51 & Thready pulse & 98 & 31.31 \\
\hline 8 & Pink tongue & 54 & 25.84 & Pulse sinking & 200 & 27.03 & Acid heavy pain & 95 & 30.35 \\
\hline 9 & Thin white tongue coating & 53 & 25.36 & White tongue coating & 191 & 25.81 & Pharynx trunk & 87 & 27.80 \\
\hline 10 & Yellow tongue coating & 52 & 24.88 & Pink tongue & 188 & 25.41 & Yellow tongue coating & 81 & 25.88 \\
\hline 11 & Bitter taste & 47 & 22.49 & Yellow tongue coatir & 180 & 24.32 & Chest tightness & 77 & 24.60 \\
\hline 12 & Fat and sweet & 47 & 22.49 & Thready pulse & 179 & 24.19 & Sleep disturbance. & 77 & 24.60 \\
\hline 13 & White tongue coating & 46 & 22.01 & Tooth-marked tongue & 179 & 24.19 & Spitting & 76 & 24.28 \\
\hline 14 & Impatient and irritable & 44 & 21.05 & Thin white tongue coating & 179 & 24.19 & Blurred vision & 72 & 23.00 \\
\hline 15 & Thirsty to drink cold & 44 & 21.05 & Lumbago & 175 & 23.65 & Insomnia & 71 & 22.68 \\
\hline 16 & Tiredness and fatigue & 43 & 20.57 & Tiredness and fatigue & 173 & 23.38 & Pulse sinking & 66 & 21.09 \\
\hline 17 & Addicted to spicy food & 42 & 20.10 & Fat and sweet & 161 & 21.76 & Rapid pulse & 65 & 20.77 \\
\hline 18 & Body fat & 41 & 19.62 & Insomnia & 157 & 21.22 & Tooth-marked tongue & 64 & 20.45 \\
\hline 19 & Acid heavy pain & 41 & 19.62 & Chest tightness & 153 & 20.68 & Lumbago & 64 & 20.45 \\
\hline 20 & Rapid pulse & 40 & 19.14 & Blurred vision & 148 & 20.00 & Thick sputum & 64 & 20.45 \\
\hline
\end{tabular}

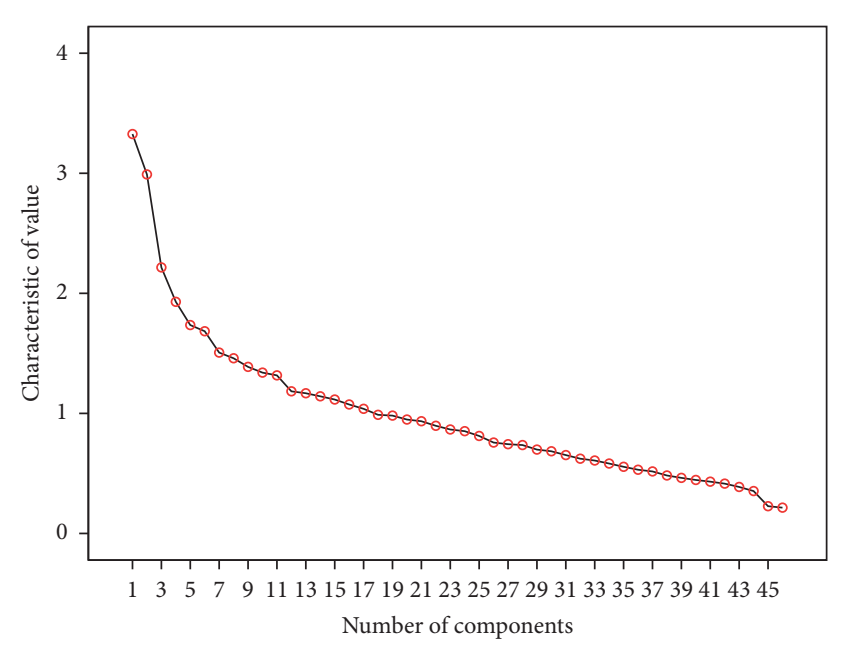

FIGURE 2: Scree plot of the characteristic root value of common factors. The number of common factors is shown on the $x$-axis and the characteristic values on the $y$-axis. Each node represents a common factor, which is arranged according to its characteristic value.

syndrome. Specifically, there were some significant differences in each group. In the elderly group, the frequency of blood deficiency and Yin deficiency syndrome was obviously higher than that in the other two groups, while the frequency of Qi deficiency syndrome was lower than that in the other groups. In the young group, the frequency of heat syndrome was obviously higher than that in the other two groups.

In the aspect of location syndrome elements (Table 5, Figure 4), the top three frequencies in the young group were heart, stomach, and spleen syndrome; in the middle-aged group, the top three frequencies were heart, liver, and kidney syndrome; and in the elderly group, the top three frequencies were liver and heart, spleen, and lung syndrome. There are also some specific significant differences among these groups. For the young group, the frequency of stomach syndrome was obviously higher than that in the other two groups. For the middle-aged group, the frequency of kidney syndrome was obviously higher than that in the elderly group. For the elderly group, the frequency of lung, gallbladder, and kidney syndrome was higher than that in the middle-aged group.

3.7. Cluster Analysis of MS Syndrome Type. Scores of 19 common factors in each age group were taken as variables in the $K$-means cluster analysis. Then, those variables were clustered to 5 final cluster centers. It can be seen from the ANOVA table (Table 6) that almost all common factors contribute to this clustering with $P<0.001$ (except for F3). The top five common factors owning the largest contribution are F2, F17, F8, F18, and F11. The CVCR of the combined factors was $20.86 \%, 15.18 \%, 10.54 \%, 10.73 \%$, and $6.90 \%$, respectively (Table 7).

Combined the cluster results with the expert's suggestions and the relevant standards of MS diagnosis in TCM, all samples were classified into five types: phlegm heat syndrome (PHS), hyperactivity of heart fire syndrome (HHFS), spleen deficiency and dampness syndrome (SDDS), deficiency of liver and kidney syndrome (DLKS), and phlegm turbid upper syndrome (PTUS). There was a significant difference in the overall distribution of syndrome types among the three groups by the $\mathrm{X}^{2}$ test (Figure 5). The top 3 frequencies of syndrome types in the three groups are spleen deficiency and dampness syndrome, phlegm and heat syndrome, and phlegm and turbid upper syndrome. The frequency of spleen deficiency and dampness syndrome in the young group was obviously higher than that in the old group. The frequency of phlegm heat syndrome in the elderly group was obviously higher than that in the middleaged group. 
TABLE 3: Syndrome elements and the target index represented by common factors.

\begin{tabular}{|c|c|c|c|}
\hline \multirow{2}{*}{$\begin{array}{l}\text { Common } \\
\text { factor }\end{array}$} & \multirow{2}{*}{ Four diagnostic variables* } & \multicolumn{2}{|c|}{ Syndrome elements } \\
\hline & & Nature & Location \\
\hline F1 & $\begin{array}{c}\text { Spitting } 0.776 \text {, thick phlegm } 0.760 \text {, sleep disturbance } 0.359 \text {, greasy tongue coating } \\
0.396 \text {, wiry pulse } 0.314 \text {, heavy body trapped } 0.422\end{array}$ & Phlegm, dampness & Lung, spleen \\
\hline F2 & Upset 0.796 , dysphoria 0.775 , impatient and irritable 0.584 & Yang hyperactivity & Heart, liver \\
\hline F3 & Yellow tongue coating 0.813 , thick tongue coating 0.652 , smoking 0.317 & Phlegm, heat & - \\
\hline F4 & Blurred vision 0.685 , dim eyesight 0.576 , dizziness 0.563 & Blood deficiency & Liver \\
\hline F5 & White tongue coating 0.804 , greasy tongue coating 0.600 , fatigue 0.309 & $\begin{array}{l}\text { Cold, dampness, Qi } \\
\text { deficiency }\end{array}$ & - \\
\hline F6 & Flustered 0.740 , palpitation 0.673 , chest distress 0.539 & - & Heart \\
\hline F7 & Lumbago 0.812 , acid heavy pain 0.801 & Dampness & Kidney \\
\hline F8 & Wiry pulse 0.654 , lip purple 0.446 , dry throat 0.355 , smoking 0.309 & $\begin{array}{l}\text { Blood stasis, Yin } \\
\text { deficiency }\end{array}$ & Liver \\
\hline F9 & Thirsty to drink heat 0.332 , thirsty 0.745 , pharynx trunk 0.619 , bitter taste 0.475 & Yin deficiency & Gallbladder \\
\hline F10 & High fat diet 0.675 , addicted to spicy food 0.660 , smoking 0.522 & Phlegm, heat & Stomach \\
\hline F11 & Epigastric distention 0.664 , heavy body trapped 0.486 & $\begin{array}{l}\text { Stagnation of Qi, } \\
\text { dampness }\end{array}$ & Spleen \\
\hline F12 & Body fat 0.798 , pink tongue 0.713 & Sputum & - \\
\hline F13 & Sleep disturbance 0.350 , dreaminess 0.767 , insomnia 0.626 & - & Heart \\
\hline F14 & Thirsty to drink heat 0.371 , slippery pulse 0.535 & Dampness & - \\
\hline F15 & Pulse sink 0.800 & In & - \\
\hline F16 & Spontaneous sweating 0.802 , thirsty to drink cold 0.475 & $\begin{array}{l}\text { Deficiency of Qi and } \\
\text { heat }\end{array}$ & - \\
\hline F17 & Thin white tongue coating 0.585 & Surface & - \\
\hline F18 & Constant fear of cold 0.768 & Yang deficiency & - \\
\hline F19 & Waist and knees are soft and sour 0.611 , frequent loose stools 0.569 & - & $\begin{array}{l}\text { Spleen, } \\
\text { Kidney }\end{array}$ \\
\hline
\end{tabular}

${ }^{*}$ Values in this table are results of the factor load matrix after rotation transformation.

TABLE 4: Distribution of nature syndrome in different age groups of MS patients.

\begin{tabular}{|c|c|c|c|c|c|c|c|c|}
\hline \multirow{2}{*}{ Nature syndrome } & \multicolumn{2}{|c|}{ Young group } & \multicolumn{2}{|c|}{ Middle-aged group } & \multicolumn{2}{|c|}{ Elderly group } & \multicolumn{2}{|c|}{ Total } \\
\hline & $N$ & $\%$ & $N$ & $\%$ & $N$ & $\%$ & $N$ & $\%$ \\
\hline Phlegm & 69 & 33.01 & 192 & 25.95 & 75 & 23.96 & 336 & 26.62 \\
\hline Dampness & 44 & 21.05 & 178 & 24.05 & 91 & 29.07 & 313 & 24.80 \\
\hline Cold & 13 & 6.22 & 49 & 6.62 & 12 & 3.83 & 74 & 5.86 \\
\hline Heat ${ }^{\mathrm{abc}}$ & 64 & 30.62 & 161 & 21.76 & 31 & 9.90 & 256 & 20.29 \\
\hline Qi stagnation & 10 & 4.78 & 34 & 4.59 & 17 & 5.43 & 61 & 4.83 \\
\hline Stasis of blood & 4 & 1.91 & 30 & 4.05 & 18 & 5.75 & 52 & 4.12 \\
\hline Yang hyperactivity & 9 & 4.31 & 41 & 5.54 & 16 & 5.11 & 66 & 5.23 \\
\hline Qi deficiency ${ }^{\text {bc }}$ & 33 & 15.79 & 103 & 13.92 & 21 & 6.71 & 157 & 12.44 \\
\hline Yang deficiency & 9 & 4.31 & 51 & 6.89 & 14 & 4.47 & 74 & 5.86 \\
\hline Yin deficiency ${ }^{b c}$ & 9 & 4.31 & 54 & 7.30 & 46 & 14.70 & 109 & 8.64 \\
\hline Blood deficiency ${ }^{\text {bc }}$ & 5 & 2.39 & 33 & 4.46 & 29 & 9.27 & 67 & 5.31 \\
\hline
\end{tabular}

${ }^{\mathrm{a}} \mathrm{A}$ significant difference between the young group and the middle-aged group. ${ }^{\mathrm{b}} \mathrm{A}$ significant difference between the young group and the elderly group. ${ }^{\mathrm{c}} \mathrm{A}$ significant difference between the elderly group and the middle-aged group.

\section{Discussion}

4.1. Multivariate Statistical Method and TCM Syndrome Differentiation. Syndrome differentiation and treatment is the essence of TCM [19]. The information collected from the four diagnostic methods (inspection, auscultation/smelling, interrogation, and palpating) is an important basis for the diagnosis and treatment of TCM. However, the TCM syndrome information has the characteristics of subjective, complex, multidimensional, multilevel, and so on, which increases the difficulty in clinical and scientific research of TCM syndrome. Factor analysis is a statistical method to find out the limited unobservable potential variables, i.e., common factors that dominate the relationship from the internal dependence of the correlation matrix of the original variables, and then explain the correlation or covariance relationship between the original variables [20,21]. Cluster analysis is used to find a kind of statistics that can objectively reflect the affinity between things and then classify things according to these statistics and some classification criteria [21], which aim at making the differences within each category as small as possible, and the differences between categories as large as possible. In TCM, the syndrome cannot be observed directly but can be reflected by a series of 


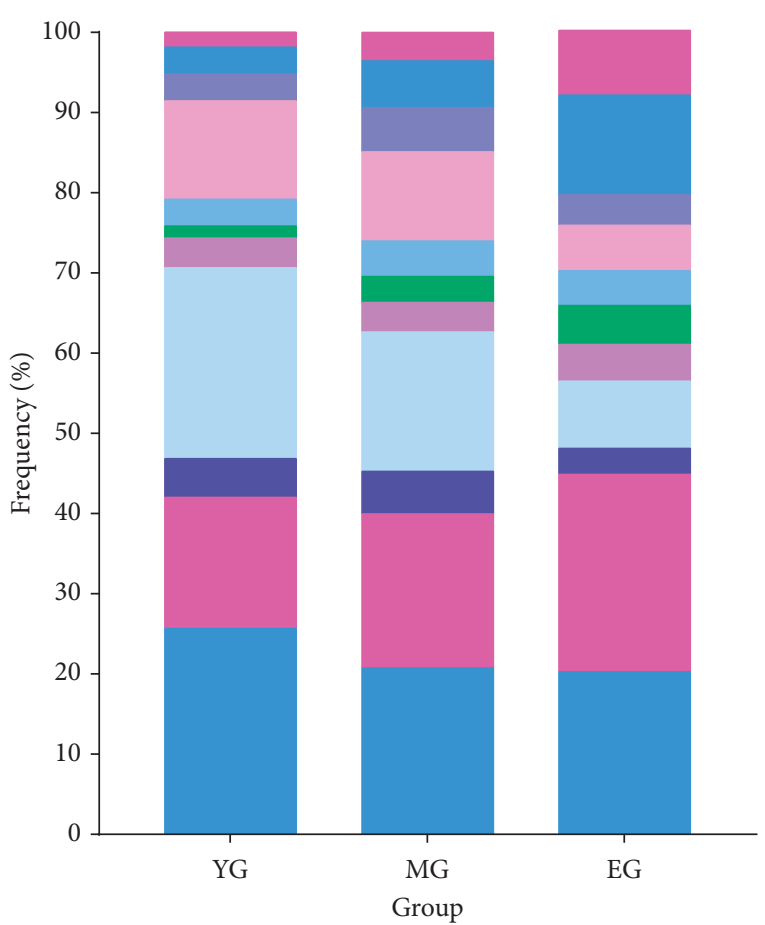

Nature syndrome elements

\begin{tabular}{|c|c|}
\hline Blood deficiency ${ }^{\mathrm{bc}}$ & Qi stagnation \\
\hline Yin deficiency $^{\mathrm{bc}}$ & Heat $^{\text {abc }}$ \\
\hline Yang deficiency & Cold \\
\hline Qi deficiency ${ }^{\text {bc }}$ & Dampness \\
\hline Yang hyperactivity & Phlegm \\
\hline Stasis of blood & \\
\hline
\end{tabular}

FIGURE 3: Distribution of nature syndrome in different age groups of MS patients. The nature syndrome element distribution of blood deficiency, Yin deficiency, Qi deficiency, and heat was obviously different among three age groups. "A significant difference between the young and the middle-aged groups; ${ }^{\mathrm{b}} \mathrm{a}$ significant difference between the young and the elderly groups; ${ }^{c}$ a significant difference between the elderly and the middle-aged groups.

TABLE 5: Distribution of location syndrome in different age groups of MS patients.

\begin{tabular}{|c|c|c|c|c|c|c|c|c|}
\hline \multirow[t]{2}{*}{$\begin{array}{l}\text { Location } \\
\text { syndrome }\end{array}$} & \multicolumn{2}{|c|}{$\begin{array}{l}\text { Young } \\
\text { group }\end{array}$} & \multicolumn{2}{|c|}{$\begin{array}{l}\text { Middle- } \\
\text { aged } \\
\text { group }\end{array}$} & \multicolumn{2}{|c|}{$\begin{array}{l}\text { Elderly } \\
\text { group }\end{array}$} & \multicolumn{2}{|c|}{ Total } \\
\hline & $N$ & $\%$ & $N$ & $\%$ & $N$ & $\%$ & $N$ & $\%$ \\
\hline Lung $^{c}$ & 9 & 4.31 & 40 & 5.41 & 36 & 11.50 & 85 & 6.74 \\
\hline Heart & 24 & 11.48 & 118 & 15.95 & 63 & 20.13 & 205 & 16.24 \\
\hline Liver & 18 & 8.61 & 104 & 14.05 & 63 & 20.13 & 185 & 14.66 \\
\hline Gallbladder $^{c}$ & 5 & 2.39 & 24 & 3.24 & 28 & 8.95 & 57 & 4.52 \\
\hline Spleen & 22 & 10.53 & 81 & 10.95 & 42 & 13.42 & 145 & 11.49 \\
\hline Stomach ${ }^{\mathrm{abc}}$ & 23 & 11.00 & 54 & 7.30 & 7 & 2.24 & 84 & 6.66 \\
\hline Kidney $^{c}$ & 19 & 9.09 & 84 & 11.35 & 22 & 7.03 & 125 & 9.90 \\
\hline
\end{tabular}

symptoms and signs that may potentially be related. The basic unit of TCM syndrome is the syndrome element, including the nature and location syndrome elements, which is similar to the common factor. Factor analysis can be used to find the potential relationship behind the four diagnosis information of TCM, namely, the common factors

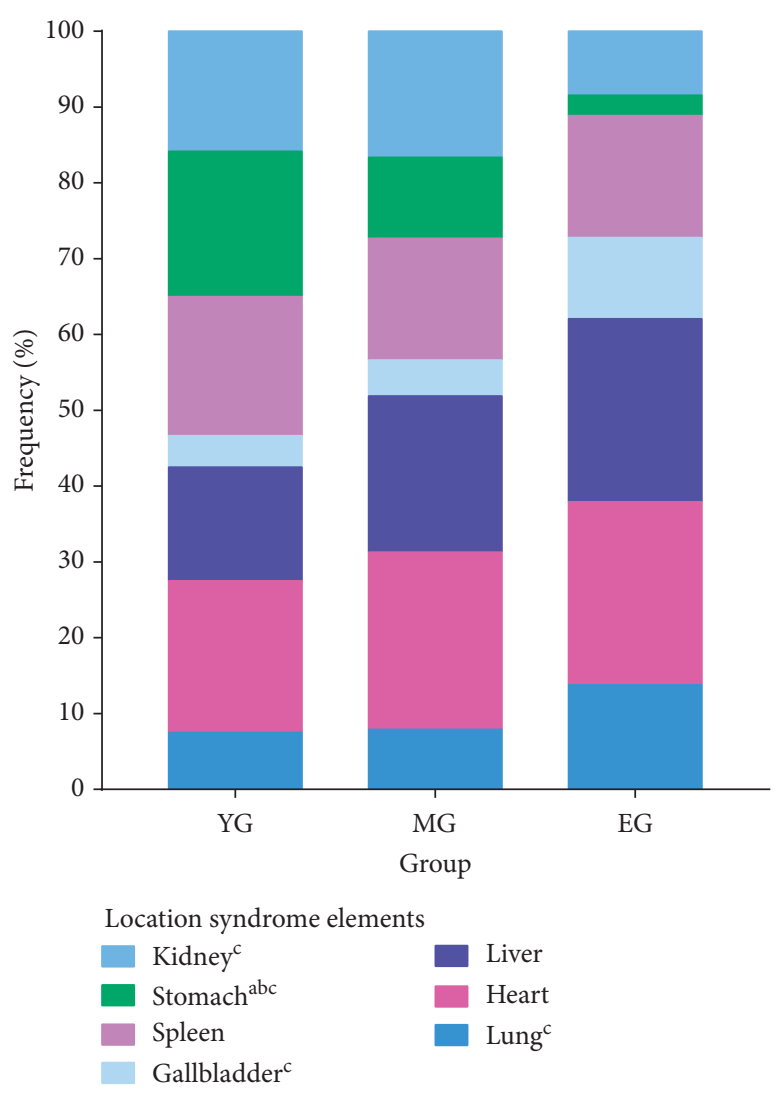

FIgURE 4: Distribution of location syndrome in different age groups of MS patients. The location syndrome distribution of the kidney, stomach, gallbladder, and lung was obviously different among three age groups.

TABLE 6: ANOVA.

\begin{tabular}{lcccccc}
\hline \multirow{2}{*}{ Factor variables } & \multicolumn{2}{c}{ Cluster } & \multicolumn{2}{c}{ Error } & $F$ & Sig. \\
& Ms & Df & Ms & Df & & \\
\hline F1 & 40.616 & 4 & 0.874 & 1257 & 46.475 & 0.000 \\
F2 & 194.800 & 4 & 0.383 & 1257 & 508.229 & 0.000 \\
F3 & 2.090 & 4 & 0.997 & 1257 & 2.097 & 0.079 \\
F4 & 28.403 & 4 & 0.913 & 1257 & 31.117 & 0.000 \\
F5 & 22.410 & 4 & 0.932 & 1257 & 24.048 & 0.000 \\
F6 & 5.472 & 4 & 0.986 & 1257 & 5.551 & 0.000 \\
F7 & 31.322 & 4 & 0.904 & 1257 & 34.667 & 0.000 \\
F8 & 58.548 & 4 & 0.817 & 1257 & 71.673 & 0.000 \\
F9 & 19.032 & 4 & 0.943 & 1257 & 20.190 & 0.000 \\
F10 & 11.051 & 4 & 0.968 & 1257 & 11.416 & 0.000 \\
F11 & 51.446 & 4 & 0.839 & 1257 & 61.283 & 0.000 \\
F12 & 6.060 & 4 & 0.984 & 1257 & 6.160 & 0.000 \\
F13 & 6.639 & 4 & 0.982 & 1257 & 6.761 & 0.000 \\
F14 & 15.807 & 4 & 0.953 & 1257 & 16.589 & 0.000 \\
F15 & 6.494 & 4 & 0.983 & 1257 & 6.610 & 0.000 \\
F16 & 44.689 & 4 & 0.861 & 1257 & 51.905 & 0.000 \\
F17 & 94.035 & 4 & 0.704 & 1257 & 133.584 & 0.000 \\
F18 & 53.126 & 4 & 0.834 & 1257 & 63.690 & 0.000 \\
F19 & 26.020 & 4 & 0.920 & 1257 & 28.271 & 0.000 \\
\hline DF
\end{tabular}

Df: degree of freedom; Ms: mean square.

(syndrome elements). The syndrome type is composed of different nature or location syndrome elements by certain rules, which is also similar to cluster analysis that can be used 
TABLE 7: The factor constitution of syndrome classification.

\begin{tabular}{|c|c|c|c|c|}
\hline Cluster & Factor combination & $\begin{array}{c}\text { CVCR } \\
(\%)\end{array}$ & $\begin{array}{l}\text { Syndrome } \\
\text { type }\end{array}$ & Main signs and symptoms \\
\hline 1 & $F 1+\mathrm{F} 3+F 6+\mathrm{F} 7+\mathrm{F} 12$ & 20.86 & PHS & $\begin{array}{l}\text { Spitting, phlegm is thick, sleep disturbance, heavy body trapped, } \\
\text { smoking, flustered, palpitation, chest distress, acid heavy pain, obesity, } \\
\text { thick or greasy tongue coating, yellow coating, wiry pulse, etc. }\end{array}$ \\
\hline 2 & $F 2+F 13$ & 10.73 & HHFS & $\begin{array}{c}\text { Upset, fidgety, impatient and irritable, sleep disturbance, dreaminess, } \\
\text { insomnia, etc. }\end{array}$ \\
\hline 3 & $F 8+\mathrm{F} 9+\mathrm{F} 11+\mathrm{F} 14+\mathrm{F} 17+\mathrm{F} 19$ & 15.18 & DLKS & $\begin{array}{l}\text { Thirsty, pharynx trunk, bitter taste, thirsty to drink heat, smoking, } \\
\text { epigastric distention, heavy body trapped, waist and knees are soft and } \\
\text { sour, frequent loose stools, wiry or slippery pulse, etc. }\end{array}$ \\
\hline 4 & $F 5+F 15+F 16+F 18$ & 10.54 & SDDS & $\begin{array}{l}\text { Tiredness and fatigue, spontaneous sweating, constant fear of cold, } \\
\text { white tongue coating, greasy tongue coating, deep pulse, etc. }\end{array}$ \\
\hline 5 & $F 4+F 10$ & 6.90 & PTUS & $\begin{array}{c}\text { High fat diet, addicted to spicy food, smoking, blurred vision, } \\
\text { dizziness, etc. }\end{array}$ \\
\hline
\end{tabular}

PHS: phlegm heat syndrome; HHFS: hyperactivity of heart fire syndrome; SDDS: spleen deficiency and dampness syndrome; DLKS: deficiency of liver and kidney syndrome; PTUS: phlegm turbid upper syndrome.

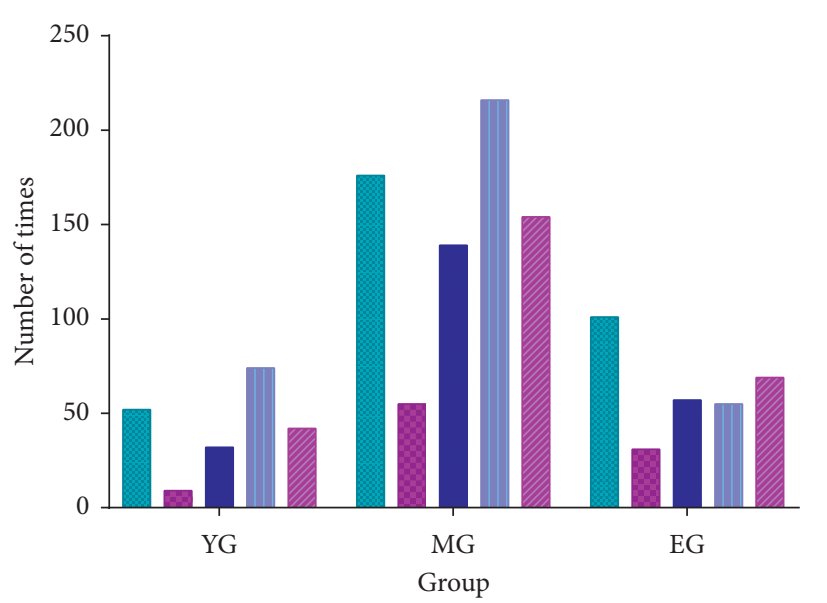

Syndrome types

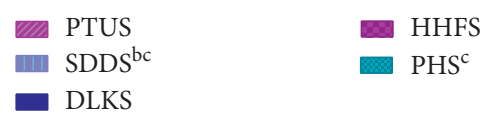

FIGURE 5: Distribution of syndrome types in different age groups of MS. The distribution of spleen deficiency and dampness syndrome and phlegm heat syndrome was obviously different among three age groups. PHS: phlegm heat syndrome; HHFS: hyperactivity of heart fire syndrome; SDDS: spleen deficiency and dampness syndrome; DLKS: deficiency of liver and kidney syndrome; PTUS: phlegm turbid upper syndrome.

to find the potential combination rules from various syndrome elements and then classify each sample into a certain syndrome type.

\subsection{The Similarities of Syndrome Distribution in MS Patient.} The occurrence and development of MS are closely related to age. Traditional Chinese medicine emphasizes "adjusting measures to individual conditions" and "treating the same disease with different methods." Despite having the same disease, patients of different ages may have different syndrome characteristics under the basic pathogenesis. Only by understanding the similarities and differences of diseases in different populations, the therapeutic effect can be improved. In this study, the common symptoms of MS, including thirst, dry throat, bitter taste, dizziness, chest distress, tiredness, flustered, impatience and irritability, lack of sleep, lumbago, blurred vision, fat body, slippery pulse or thin pulse, and white or yellow greasy coating, revealed the basic pathogenesis of excessiveness complicated with deficiency syndrome. The symptoms such as spitting, impatience, irritability, and slippery pulse indicate excess syndrome, and the dizziness, blurred vision, and tiredness suggest deficiency syndrome. Moreover, phlegm and dampness are the main pathological features in all age groups.

In clinical practice, it is found that most of the patients with metabolic syndrome are obese. According to the TCM theory, fat people are more likely to develop a phlegmdampness constitution, so they have symptoms such as spitting, feeling body heavy and trapped, and greasy tongue coating. While phlegm-dampness is prone to hurting the spleen and hindering the movement of Qi and blood, so the patient may have symptoms such as epigastric distention, lassitude and weakness, spontaneous sweating, and dizziness. With diseases lasting for a long time, Qi stagnation and blood stasis will turn out, so symptoms such as bitter taste, impatience and irritability, purple lips, and wiry pulse can be found. Moreover, the phlegm-dampness can turn into heat with time going by, and pathogenic heat can also disturb the heart, which will induce symptoms of thirst, upset, fidgety, dreaminess, insomnia, and so on. When phlegm and blood stasis combine, MS would further develop and the symptoms of viscera deficiency appear, such as blurred vision, palpitation, waist and knee soft, and often loose stools. So, on the basis of statistical analysis and professional knowledge, we ended up with five syndrome types, including phlegm heat syndrome, hyperactivity of heart fire syndrome, spleen deficiency and dampness syndrome, deficiency of liver and kidney syndrome, and phlegm turbid upper syndrome.

4.3. The Differences of Syndrome Distribution in Different Age Groups of MS. Based on the commonness of MS, there are also some different characteristics among the young, middle- 
aged, and elderly group. Compared with the middle-aged group and elderly group, the young group has more obvious nature syndrome elements in heat and Qi deficiency, and location syndrome element in the stomach. It may be related to that the young people are just at the time of excess blood and Qi, and the excess of Qi can lead to heat syndrome, just like the famous TCM doctor Danxi Zhu said, "excess of Qi is fire." So, young patients are more likely to have the heat syndrome compared to old patients. Heat evil is more likely to consume body fluid and Qi. Besides, an unhealthy diet style may easily damage Qi in spleen, so the young patients also have a higher frequency in Qi deficiency. As for the middle-aged group, the most obvious problem is that the frequency in kidney syndrome was higher than that in other groups. This may be because most patients in the middleaged group were female patients and about 50 years old. At this time, they are just in perimenopause and appear more kidney deficiency problems [22]. When it comes to the elderly group, the mechanisms of MS and its syndrome features turn out to be more complicated. According to the results, it is shown that the symptoms of Yin deficiency and blood deficiency increase with age and those old patients may have more location syndrome elements in the lung and gallbladder. So, we can also find that the elderly people have a higher frequency of symptoms such as dizziness, bitter taste, spitting, and dry throat. The results of cluster analysis show the relative difference is not big, but from it, we also find that the young and middle-aged groups have a higher frequency in spleen deficiency and dampness syndrome and phlegm heat syndrome, while the elderly group has a higher frequency in phlegm heat syndrome and phlegm turbid upper syndrome.

4.4. Deficiency and Prospect. This research is based on the real-world investigation, and the results basically conform to the syndrome distribution feature of MS in the clinic. However, there are also some differences with the previous research, which may be affected by several factors. First of all, there are some differences in the sample size of the three age groups in this study, which may have something to do with disease characteristics. Secondly, the regional environment can also affect the study results. These cases were collected in Fujian, where the climate is mostly humid and heat, which may have an impact on the frequency statistics of dampness and heat syndrome in this study. In addition, due to the limitation of statistical methods, there are some deviation indexes in the classification results, which are slightly different from other indexes in the same category.

However, in a word, factor and cluster analysis methods in this study were processed according to the internal laws of the data themselves, which reduced the errors caused by subjective judgment and revealed the syndrome characteristics of MS in different age groups from different perspectives. In this way, it can provide a certain basis for the quantitative and qualitative syndrome differentiation and treatment of MS. In future research, a large-scale and multicenter epidemiological investigation can be combined. Also, the collection process of four diagnosis information needs to be more standardized and better-optimized analysis models are supposed to make it more suitable for the clinical characteristics of TCM.

\section{Conclusion}

From the above analysis, we can find that the TCM syndrome of MS is complex and there may have some syndrome elements that appeared simultaneously. However, it is not hard to find that the main pathological factors in MS were inseparable from phlegm and dampness. Besides, MS patients at different age periods have their own syndrome distribution characteristics. Therefore, in the clinical diagnosis and treatment process, we are supposed to focus on the syndrome feature in different age stages and give consideration to the primary and secondary aspects as well as the deficiency and excess aspects. So, it is reasonable to adhere to the method of resolving phlegm and removing dampness and, at the same time, adopt the ways of clearing heat, promoting Qi, nourishing Yin, supplementing blood as well as regulating liver, promoting lung, transporting spleen, nourishing heart, and nourishing kidney based on the syndrome feature of MS in different age stages.

\section{Data Availability}

The data used to support the findings of this study are available from the corresponding author upon request.

\section{Ethical Approval}

The study was approved by the Ethics Committee of the Fujian University of Traditional Chinese Medicine (FTCMER 2012. 004).

\section{Consent}

All patients signed informed consent.

\section{Conflicts of Interest}

The authors declare that they have no conflicts of interest.

\section{Authors' Contributions}

All authors contributed to this study. SJX, CDL, and BZG conceived and designed the experiments. SJX and SJC performed the experiments. JXC and JC contributed to statistical analysis of the data. SJX and XJL wrote the manuscript. All authors have read and approved the manuscript for publication.

\section{Acknowledgments}

This work was partially supported by the Joint Fund Project of the National Natural Science Foundation of China (U1705286), National Natural Science Foundation of China (81873234), and Major Project of Standardization of the State Administration of Traditional Chinese Medicine of the PRC (GZY-FJS-2017-64). 


\section{References}

[1] E. McCracken, M. Monaghan, and S. Sreenivasan, "Pathophysiology of the metabolic syndrome," Clinics in Dermatology, vol. 36, no. 1, pp. 14-20, 2018.

[2] A. S. Hirose, K. Fujihara, F. Miyamasu et al., "Development and evaluation of the Japanese version of the audit of diabetesdependent quality of life for patients with diabetes," Diabetology International, vol. 7, no. 4, pp. 384-390, 2016.

[3] H. J. Guo, X. Nian, Y. F. Liang et al., "The prevalence and risk factors of metabolic syndrome in Chinese population based on the multi center crosssectional survey," Chinese Journal of Disease Control \& Prevention, vol. 23, no. 7, pp. 384-390, 2019.

[4] L. H. Liu, H. Q. Li, and Y. C. Xu, "Relationship between the age of onset of newly diagnosed type 2 diabetes and metabolic syndrome," Chinese General Practice, vol. 22, no. 22, pp. 2688-2691, 2019.

[5] B. Li and X. F. Jiang, "Research progress of metabolic syndrome," International Journal of Laboratory Medicine, vol. 39, no. 24, pp. 2996-3001, 2018.

[6] Y. Liu, M. Sun, H. Yao, Y. Liu, and R. Gao, "Herbal medicine for the treatment of obesity: an overview of scientific evidence from 2007 to 2017," Evidence-Based Complementary and Alternative Medicine, vol. 2017, Article ID 8943059, 17 pages, 2017.

[7] Q. Wang and L. Liu, "Advances in the treatment of metabolic syndrome by traditional Chinese medicine," Information on Traditional Chinese Medicine, vol. 34, no. 6, pp. 142-145, 2017.

[8] W. S. Zhang, A. Pan, L. Yang et al., "American ginseng and asian ginseng intervention in diet-induced obese mice: metabolomics reveals distinct metabolic profiles," The American Journal of Chinese Medicine, vol. 47, no. 4, pp. 1-15, 2019.

[9] Y. Wu, F. Zhang, K. Yang et al., "SymMap: an integrative database of traditional Chinese medicine enhanced by symptom mapping," Nucleic Acids Research, vol. 47, no. D1, pp. 1110-1117, 2019.

[10] G. Zhang, Y. H. Huang, and X.-B. Zhang, "Deep feature learning based clustering with application to TCM data analysis," in Proceedings of the 2018 9th International Conference on Information Technology in Medicine and Education (ITME), Hangzhou, China, October 2018.

[11] M. L. Zhang and Z. H. Zhou, "A $k$-nearest neighbor based algorithm for multi-label classification," in Proceedings of the IEEE International Conference on Granular Computing, Beijing, China, July 2005.

[12] H. Kang, Y. Zhao, C. Li et al., "Integrating clinical indexes into four-diagnostic information contributes to the Traditional Chinese Medicine (TCM) syndrome diagnosis of chronic hepatitis B," Scientific Reports, vol. 5, no. 1, p. 9395, 2015.

[13] Q. Xu, W. Tang, F. Teng et al., "Intelligent syndrome differentiation of traditional Chinese medicine by ANN: a case study of chronic obstructive pulmonary disease," IEEE Access, vol. 7, pp. 76167-76175, 2019.

[14] Z. Y. Ma, Z. C. Zhang, X. Sheng et al., "Study on the components of metabolic syndrome and TCM syndrome elements," Guiding Journal of Traditional Chinese Medicine and Pharmacology, vol. 25, no. 10, pp. 50-55, 2019.

[15] J. Liu, B. X. Chen, H. M. Wang et al., "Study on the combination of syndrome elements of metabolic syndrome with carotid atherosclerosis based on cluster analysis," Beijing Journal of Traditional Chinese Medicine, vol. 37, no. 9, pp. 807-810, 2018.
[16] T. S. State Bureau, National Standard of the People's Republic of China: Syndrome Part in TCM Clinical Diagnosis and Treatment Terminology, China Standards Press, Beijing, China, 1997.

[17] C. D. Li, Diagnostics of Traditional Chinese Medicine, China traditional Chinese Medicine Press, Beijing, China, 2016.

[18] Z. W. Feng, Syndrome Elements Differentiation, People's Health Press, Beijing, China, 2008.

[19] T. T. Deng, "Syndrome differentiation and treatment is the essence of traditional Chinese Medicine," Chinese Archives of Traditional Chinese Medicine, vol. 20, no. 4, pp. 394-395, 2002.

[20] Z. Q. Sun, Medical Statistics, People's Health Press, Beijing, China, 2014.

[21] W. S. Wang Jun and Z. Deng, "Some problems in cluster analysis," Control and Decision, vol. 27, no. 3, pp. 321-328, 2012.

[22] S. L. Cui, P. Zhang, W. F. Gu et al., "Literature research on traditional Chinese medicine syndrome distribution and medication rule of perimenopausal syndrome," Journal of Traditional Chinese Medicine, vol. 60, no. 22, pp. 1968-1971, 2019. 